\title{
Editorial: ImmunoPhysics and ImmunoEngineering
}

\begin{abstract}
Jorge Bernardino de la Serna ${ }^{1 *}$, Mario Mellado ${ }^{2}$, Michael L. Dustin ${ }^{3}$, Maria F. Garcia-Parajo ${ }^{4,5}$ and Dimitrios Morikis ${ }^{6 t}$

${ }^{1}$ Faculty of Medicine, National Heart and Lung Institute, Imperial College London, London, United Kingdom, ${ }^{2}$ Chemokine Signaling Group, Department of Immunology and Oncology, Centro Nacional de Biotecnologia (CNB-CSIC), Madrid, Spain, ${ }^{3}$ Kennedy Institute of Rheumatology, NDORMS-Nuffield Department of Orthopaedics, Rheumatology and Musculoskeletal Sciences, The University of Oxford, Oxford, United Kingdom, ${ }^{4}$ ICFO-Institut de Ciencies Fotoniques, The Barcelona Institute of Science and Technology, Barcelona, Spain, ${ }^{5}$ ICREA-Catalan Institution for Research and Advanced Studies, Barcelona, Spain, ${ }^{6}$ Department of Bioengineering, University of California, Riverside, Riverside, CA, United States
\end{abstract}

Keywords: immunophysics, immunoengineering, $\mathrm{T}$ cell biology, biophysics, cell membrane, cell sensing and remodeling, super resolution (SR), quantitative imaging

\section{Editorial on the Research Topic}

\section{ImmunoPhysics and ImmunoEngineering}

The immune system comprises a collection of specialized cells, tissues, and organs that protect the organisms against pathogens and can survey cancer cells. Immune responses are precisely coordinated events that take place in complex, specialized tissue microenvironments. For an integrated view of innate and adaptive immune responses at the molecular level, we ideally need a better understanding of how immune cells communicate and fulfill their tasks in vivo, following events spatially and temporally. Conventional biochemical and genetic methods consider the cell as an individual entity and ligand/receptor pairs as isolated systems. Often, the data obtained refers to the average behavior of a pool of cells and/or receptors removed from their real-life context. The use of new technologies, particularly real-time imaging approaches, is showing us that biological responses are very dynamic and extremely dependent on the context in which they take place and are therefore much more diverse than we initially thought. The combination of these new approaches is radically transforming and enriching immunology, as demonstrated by the increasing number of publications in which physical and/or engineering tools are applied to study the immune response. Whilst scientists are often questioned for the discipline their research is best framed in, we rather think that one scientific discipline cannot be reduced to the terms of another. However, defining and naming cross-disciplinary fields sets our minds on common ground and helps establish a fluent communication to eventually produce groundbreaking, beautiful pieces of science. For instance, ImmunoPhysics was probably first coined by Prof. Morikis a couple of decades ago (https://www.biophysics.org/profiles/dimitrios-morikis); nevertheless, ImmunoPhysics has not become widely regarded as a discipline, despite the continuously growing body of research that requires physical approaches to resolving immunological questions. Hence, with this special issue, we wanted to open a scientific platform compiling ImmunoPhysics and ImmunoEngineering research breakthroughs and future perspectives. Sadly, towards the end of this fascinating journey Prof. Morikis passed away; thus now, with this special issue, we would also like to pay tribute to his fundamental contributions to the field.

The qualitative and quantitative knowledge-advancement resulting from the application of physical and engineering methods and techniques in immunology is unarguable. Coordinated advances in physics and engineering technologies are revolutionizing our research strategies, contributing qualitatively, and quantitatively to breakthroughs in the understanding of immune system function and its regulation in health and disease. Probably, the overarching goals 
of ImmunoPhysics and ImmunoEngineering are to facilitate the development of therapeutic interventions to more precisely modulate and control the compromised immune response during diseases; but whereas ImmunoPhysics study and assess the physical basis of the immune response, ImmunoEngineering pursues its control and prediction. Possibly the greatest challenge for the growth of these fields is the establishment of fluent communication between physicists or engineers and immunologists. For this purpose, in this special issue, we aimed to provide a broad and interdisciplinary forum for researchers to present their personal views on the field, point to future challenges, and show their latest empirical or theoretical observations or method developments, with biomedical implications in vivo, in vitro, and in silico. Initially, we eagerly sought contributions employing highly advanced static or dynamic quantitative techniques spanning from conventional to super- or ultra-resolved microscopy in space and time to unravel protein clustering [1-8] or to disentangle the involvement of actin cortex dynamics and its mechanics in T-cell activation and synapse formation [9-15]; we also welcomed contributions applying steady and non-equilibrium quantitative fluorescence spectroscopic approaches to resolve receptor protein oligomerization and lipid-protein interactions and dynamics $[16,17]$ that can be applied to resolve immunerelevant events [18, 19]. Investigations employing systems immunology approaches, including quantitative and highthroughput assessment of the immune status and mathematical modeling of immune interactions [20-25] were also sought after; finally, we were also keen to include breakthroughs in the design or utilization of engineered tools and methods with applications to T-cell biology or immunotherapy [26-31].

Overall, we were gratefully surprised by the broad scope of approaches we were exposed to. For instance, in relation to the application of biophysical tools, including advanced light microscopy and electron microscopy methods, to better understand the molecular mechanisms of immuno-biological processes, we received two reviews: one describing the most used fluorescent microscopy approaches dealing with the polarized secretory intracellular traffic during the immune synapse (Calvo and Izquierdo), and another describing how techniques able to resolve elements at the nanoscale have contributed to the fundamental understanding of the immune synapse (Shannon and Owen). We also received three original articles, one showing a new developed method to track soluble mediators in the B-follicle at the single-molecule level (Miller et al.), another about possible artifacts occurring during chemical fixation in $\mathrm{T}$ cells (Pereira et al.), and another revealing novel structures in the podosomes formed at the dendritic cells employing correlative light and electron super-resolution microscopy (Joosten et al.). Regarding the use of in silico simulations and mathematical modeling of immune interactions and quantitative approaches to resolving lymphocyte dynamic immune-related events, we received four contributions: one resolving a structural model for the transmembrane domain of the B-cell receptor with molecular simulation (Friess et al.), another integrating experiments and theory to better understand the dynamics of the T-cell receptor and the cognate peptide of the major histocompatibility complex (Buckle and Borg), a third employing molecular dynamics and electrostatic studies to evaluate the importance of two proteins of the membrane attack complex in the initial steps of the formation of the complement system (Zewde et al.), and a fourth employing mathematical models to predict the fate of a naïve $\mathrm{T}$ cell during migration and recirculation (de la Higuera et al.). We also received articles dealing with the study of the cognate interaction between antigen-presenting cells and $\mathrm{T}$ cells, where the roles of different proteins were investigated, such as the protein kinase $\mathrm{C}$ during polarized exosome secretion (Herranz et al.), TCR interplay with CD3/CD6 (Meddens et al.), and with CD28 (Xia et al.) during the immune synapse. We also include reviews dealing with the plastic cellular morphological changes during early activation, upon triggering, and during the immune synapse, involving perspectives spanning from the highly dynamic, very sophisticated, and complex reorganization of receptor kinases and phosphatases at the plasma membrane (Pérez-Ferreros et al.; Glatzová and Cebecauer; Junghans et al.), and its associated lipids (Baumgart et al.; Pettmann et al.; Gawden-Bone and Griffiths) to the full cytoskeletal reassembly (Martín-Cófreces and Sánchez-Madrid.) The current increasing interest in the role of mechanotransduction in T-cell biology also received attention, with contributions dealing with the application of different biophysical tools to reveal cellular forces or molecular sensing to understand the correlation between the highly coordinated forces exerted by cells with their chemical sensing and activation processes (Kolawole et al.; Harrison et al.; Brockman and Salaita; Rossy et al.). Also, some contributions showed how the application of systems immunology and protein and lipid engineering could help predict lymphocyte receptor recognition patterns (Hammerschmidt et al.; Gorby et al.; Nieto-Garai et al.; Hörner et al.). Finally, contributions are also included on how building sophisticated mimetic in vitro models, for instance by means of microfluidics, novel substrates, and hydrogels, can help to unravel the complexity of immune molecular events at the single-cell level (Benard et al.; Sinha et al.; Weiden et al.).

Overall, the greatest reward was to continuously receive contribution requests, even after the closure of the submission period and to observe that the number of manuscript views and downloads is continuously increasing. We truly believe that, thanks to the 30 contributions comprising this Research Topic, we have fulfilled our aim of editing an article collection that stimulates and facilitates a scientific forum for physicists, engineers, and immunologists, filling the cross-disciplinary gap, increasing awareness, and maximizing discussion and dissemination of ideas and methodologies in this nascent field. The next challenges in the ImmunoPhysics and ImmunoEngineering fields will probably be related to mechanobiology, immuno-oncotherapy, and the engineering of the tumor immunological niche. Correlating the highly coordinated forces exerted by cells with their chemical sensing and activation process is seemingly very challenging but eventually scientifically rewarding. Furthermore, immunotherapy is now widely considered to be one of the best approaches to treatments for certain types 
of advanced cancer, and immune evasion is now recognized as a hallmark of cancer. The physical understanding of the immunological synapse underpins checkpoint therapies, and immunoengineering of T-cell specificity is the basis of CAR-T therapies. However, there is still much to learn about the physical mechanisms that differentiate responders and non-responders to these powerful therapies. We still have much to learn about engineering the tumor immunological microenvironment. One future goal of ImmunoPhysics could be to construct an accurate in vitro model tissue environment, where advanced microscopy and spectroscopy, force microscopy, and eventually optical and magnetic tweezers would enable measurement and manipulation of key physical parameters to improve

\section{REFERENCES}

1. Rossy J, Owen DM, Williamson DJ, Yang Z, Gaus K. Conformational states of the kinase Lck regulate clustering in early T cell signaling. Nat Immunol. (2012) 14:82-9. doi: 10.1038/ni.2488

2. Williamson DJ, Owen DM, Rossy J, Magenau A, Wehrmann M, Gooding JJ, et al. Pre-existing clusters of the adaptor Lat do not participate in early T cell signaling events. Nat Immunol. (2011) 12:655-62. doi: 10.1038/ni.2049

3. Santos AM, Ponjavic A, Fritzsche M, Fernandes RA, De La Serna JB, Wilcock MJ, et al. Capturing resting T cells: the perils of PLL. Nat Immunol. (2018) 19:203-5. doi: 10.1038/s41590-018-0048-8

4. Bakker GJ, Eich C, Torreno-Pina JA, Diez-Ahedo R, Perez-Samper G, Van Zanten TS, et al. Lateral mobility of individual integrin nanoclusters orchestrates the onset for leukocyte adhesion. Proc Natl Acad Sci USA. (2012) 109:4869-74. doi: 10.1073/pnas.1116425109

5. Calebiro D, Rieken F, Wagner J, Sungkaworn T, Zabel U, Borzi A. Singlemolecule analysis of fluorescently labeled G-protein-coupled receptors reveals complexes with distinct dynamics and organization. Proc Natl Acad Sci USA. (2013) 110:743-8. doi: 10.1073/pnas.1205798110

6. Fujiwara TK, Iwasawa K, Kalay Z, Tsunoyama TA, Watanabe Y, Umemura $\mathrm{YM}$, et al. Confined diffusion of transmembrane proteins and lipids induced by the same actin meshwork lining the plasma membrane. Mol Biol Cell. (2016) 27:1101-19. doi: 10.1091/mbc.E15-04-0186

7. Martínez-Muñoz L, Rodríguez-Frade JM, Barroso R, Sorzano CÓS, Torreño-Pina JA, Santiago CA, et al. Separating actin-dependent chemokine receptor nanoclustering from dimerization indicates a role for clustering in CXCR4 signaling and function. Mol Cell. (2018) 71:873. doi: 10.1016/j.molcel.2018.08.012

8. Sungkaworn T, Rieken F, Lohse MJ, Calebiro D. High-resolution spatiotemporal analysis of receptor dynamics by single-molecule fluorescence microscopy. J Vis Exp. (2014) 89:e51784. doi: 10.3791/51784

9. Ashdown GW, Burn GL, Williamson DJ, PandŽić E, Peters R, Holden M, et al. Live-cell super-resolution reveals F-actin and plasma membrane dynamics at the $\mathrm{T}$ cell synapse. Biophys J. (2017) 112:1703-13. doi: 10.1016/j.bpj.2017.01.038

10. Colin-York H, Kumari S, Barbieri L, Cords L, Fritzsche M. Distinct actin cytoskeleton behaviour in primary and immortalised T-cells. J Cell Sci. (2020) 133:jcs232322. doi: 10.1242/jcs. 232322

11. Fritzsche M, Fernandes RA, Chang VT, Colin-York H, Clausen MP, Felce $\mathrm{JH}$, et al. Cytoskeletal actin dynamics shape a ramifying actin network underpinning immunological synapse formation. Sci Adv. (2017) 3:e1603032. doi: $10.1126 /$ sciadv.1603032

12. Gawden-Bone CM, Frazer GL, Richard AC, Ma CY, Strege K, Griffiths GM. PIP5 kinases regulate membrane phosphoinositide and actin composition for targeted granule secretion by cytotoxic lymphocytes. immunity. (2018) 49:427-37.e4. doi: 10.1016/j.immuni.2018. 08.017

13. Hons M, Kopf A, Hauschild R, Leithner A, Gaertner F, Abe J, et al. Chemokines and integrins independently tune actin flow and substrate fundamental understanding, ultimately leading to applications in improved immunotherapies.

\section{AUTHOR CONTRIBUTIONS}

All authors listed have made a substantial, direct and intellectual contribution to the work, and approved it for publication.

\section{ACKNOWLEDGMENTS}

We would like to thank the reviewers for their thoughtful comments and efforts toward improving the manuscripts of this special issue. friction during intranodal migration of T cells. Nat Immunol. (2018) 19:60616. doi: 10.1038/s41590-018-0109-Z

14. Ritter AT, Kapnick SM, Murugesan S, Schwartzberg PL, Griffiths GM, Lippincott-Schwartz J. Cortical actin recovery at the immunological synapse leads to termination of lytic granule secretion in cytotoxic T lymphocytes. Proc Natl Acad Sci USA. (2017) 114: E6585-94. doi: 10.1073/pnas.1710751114

15. Valitutti S, Dessing M, Aktories K, Gallati H, Lanzavecchia A. Sustained signaling leading to $\mathrm{T}$ cell activation results from prolonged $\mathrm{T}$ cell receptor occupancy. Role of T cell actin cytoskeleton. J Exp Med. (1995) 181:577-84. doi: 10.1084/jem.181.2.577

16. Garcia E, Bernardino De La Serna J. Dissecting single-cell molecular spatiotemporal mobility and clustering at focal adhesions in polarised cells by fluorescence fluctuation spectroscopy methods. Methods. (2018) 140-41:8596. doi: 10.1016/j.ymeth.2018.03.008

17. Schneider F, Waithe D, Galiani S, Bernardino De La Serna J, Sezgin E, Eggeling C. Nanoscale spatiotemporal diffusion modes measured by simultaneous confocal and stimulated emission depletion nanoscopy imaging. Nano Lett. (2018) 18:4233-40. doi: 10.1021/acs.nanolett.8b01190

18. Hedde PN, Staaf E, Singh SB, Johansson S, Gratton E. Pair correlation analysis maps the dynamic two-dimensional organization of natural killer cell receptors at the synapse. ACS Nano. (2019) 13:14274-82. doi: 10.1021/acsnano.9b07486

19. McCloskey MA, Poo MM. Contact-induced redistribution of specific membrane components: local accumulation and development of adhesion. $J$ Cell Biol. (1986) 102:2185-96. doi: 10.1083/jcb.102.6.2185

20. Dritschel H, Waters SL, Roller A, Byrne HM. A mathematical model of cytotoxic and helper $\mathrm{T}$ cell interactions in a tumour microenvironment. Lett Biomath. (2018) 5:1-33. doi: 10.1080/23737867.2018.1465863

21. Freeman GJ, Long AJ, Iwai Y, Bourque K, Chernova T, Nishimura $H$, et al. Engagement of the PD-1 immunoinhibitory receptor by a novel B7 family member leads to negative regulation of lymphocyte activation. J Exp Med. (2000) 192:1027-34. doi: 10.1084/jem.192.7.1027

22. Mahlbacher GE, Reihmer KC, Frieboes HB. Mathematical modeling of tumor-immune cell interactions. J Theoret Biol. (2019) 469:47-60. doi: 10.1016/j.jtbi.2019.03.002

23. Polley A, Orłowski A, Danne R, Gurtovenko AA, Bernardino De La Serna J, Eggeling C, et al. Glycosylation and lipids working in concert direct CD2 ectodomain orientation and presentation. J Phys Chem Lett. (2017) 8:1060-66. doi: 10.1021/acs.jpclett.6b02824

24. Qi SY, Groves JT, Chakraborty AK. Synaptic pattern formation during cellular recognition. Proc Natl Acad Sci USA. (2001) 98:6548-53. doi: 10.1073/pnas.111536798

25. Rosenberg EM,Harrison RES, Tsou LK, Drucker N, Humphries B, Rajasekaran $\mathrm{D}$, et al. Characterization, dynamics, and mechanism of CXCR4 antagonists on a constitutively active mutant. Cell Chem Biol. (2019) 26:662-73.e7. doi: 10.1016/j.chembiol.2019.01.012

26. Caliendo F, Dukhinova M, Siciliano V. Engineered cell-based therapeutics: synthetic biology meets immunology. Front Bioeng Biotechnol. (2019) 7:43. doi: $10.3389 /$ fbioe. 2019.00043 
27. Compte M, Harwood SL, Muñoz IG, Navarro R, Zonca M, Perez-Chacon G, et al. A tumor-targeted trimeric 4-1BB-agonistic antibody induces potent anti-tumor immunity without systemic toxicity. Nat Commun. (2018) 9:4809. doi: 10.1038/s41467-018-0 7195-w

28. Krummel MF, Allison JP. Cd28 and Ctla-4 have opposing effects on the response of t-cells to stimulation. J Exp Med. (1995) 182:459-65. doi: 10.1084/jem.182.2.459

29. Roh KH. Artificial methods for $\mathrm{T}$ cell activation: critical tools in $\mathrm{T}$ cell biology and $\mathrm{t}$ cell immunotherapy. Adv Exp Med Biol. (2018) 1064:207-19. doi: 10.1007/978-981-13-044 5-3_13

30. Wu C-Y, Rupp LJ, Roybal KT, Lim WA. Synthetic biology approaches to engineer $\mathrm{T}$ cells. Curr Opin Immunol. (2015) 35:123-30. doi: $10.1016 /$ j.coi.2015.06.015
31. Li D, Li X, Zhou W-L, Huang Y, Liang X, Jiang L, et al. Genetically engineered T cells for cancer immunotherapy. Signal Transduct Target Ther. (2019) 4:35. doi: 10.1038/s41392-019-0070-9

Conflict of Interest: The authors declare that the research was conducted in the absence of any commercial or financial relationships that could be construed as a potential conflict of interest.

Copyright (c) 2020 Bernardino de la Serna, Mellado, Dustin, Garcia-Parajo and Morikis. This is an open-access article distributed under the terms of the Creative Commons Attribution License (CC BY). The use, distribution or reproduction in other forums is permitted, provided the original author(s) and the copyright owner(s) are credited and that the original publication in this journal is cited, in accordance with accepted academic practice. No use, distribution or reproduction is permitted which does not comply with these terms. 